\title{
Clinical Use of High-Intensity Focused Ultrasound (HIFU) for Tumor and Pain Reduction in Advanced Pancreatic Cancer
}

\section{Klinischer Einsatz des hoch-intensiven fokussierten Ultraschalls (HIFU) zur Tumor- und Schmerzreduktion bei fortgeschrittenem Pankreas- karzinom}

Authors

Affiliations
H. M. Strunk ${ }^{1 \S}$, J. Henseler ${ }^{1 \S}$, M. Rauch¹, M. Mücke², 3, 4, G. Kukuk¹, H. Cuhls ${ }^{2}$, L. Radbruch ${ }^{2}$, L. Zhang ${ }^{5}$, H. H. Schild ${ }^{1}$, M. Marinova

Affiliation addresses are listed at the end of the article.

\section{Key words}

- pancreatic carcinoma

$\checkmark$ high-intensity focused ultrasound

- tumor ablation

- pain relief

- cancer pain received 3.1.2016 accepted $\quad 15.3 .2016$

\section{Bibliography}

DOI http://dx.doi.org/ 10.1055/s-0042-105517 Published online: 7.6.2016 Fortschr Röntgenstr 2016; 188: 662-670 @ Georg Thieme Verlag KG Stuttgart - New York . ISSN 1438-9029

\section{Correspondence}

Milka Marinova, PhD, MD

Department of Radiology, Hospital \& Medical School, University of Bonn

Siegmund-Freud-Str. 25

D-53105 Bonn

Germany

Tel.: ++49/2 28/28711017

Fax: ++49/2 28/2 87/9011017 milka.marinova@ukb.uni-bonn. de

\section{Abstract \\ $\nabla$}

Purpose: Evaluation of ultrasound-guided high-intensity focused ultrasound (HIFU) used for the first time in Germany in patients with inoperable pancreatic cancer for reduction of tumor volume and relief of tumor-associated pain.

Materials and Methods: 15 patients with locally advanced inoperable pancreatic cancer and tumor-related pain symptoms were treated by HIFU ( $n=6$ UICC stage III, $n=9$ UICC stage IV). 13 patients underwent simultaneous standard chemotherapy. Ablation was performed using the JC HIFU system (Chongqing, China HAIFU Company) with an ultrasonic device for real-time imaging. Imaging follow-up (US, CT, MRI) and clinical assessment using validated questionnaires (NRS, BPI) was performed before and up to 15 months after HIFU. Results: Despite biliary or duodenal stents (4/ 15) and encasement of visceral vessels (15/ 15), HIFU treatment was performed successfully in all patients. Treatment time and sonication time were $111 \mathrm{~min}$ and $1103 \mathrm{~s}$, respectively. The applied total energy was $386768 \mathrm{~J}$. After HIFU ablation, contrast-enhanced imaging showed devascularization of treated tumor regions with a significant average volume reduction of $63.8 \%$ after 3 months. Considerable pain relief was achieved in 12 patients after HIFU (complete or partial pain reduction in 6 patients).

Conclusion: US-guided HIFU with a suitable acoustic pathway can be used for local tumor control and relief of tumor-associated pain in patients with locally advanced pancreatic cancer.

\footnotetext{
$\S$ contributed equally.
}

Key points:

- US-guided HIFU allows an additive treatment of unresectable pancreatic cancer.

- HIFU can be used for tumor volume reduction.

- Using HIFU, a significant reduction of cancer-related pain was achieved.

- HIFU provides clinical benefit in patients with pancreatic cancer.

Citation Format:

- Strunk HM, Henseler J, Rauch M et al. Clinical Use of High-Intensity Focused Ultrasound (HIFU) for Tumor and Pain Reduction in Advanced Pancreatic Cancer. Fortschr Röntgenstr 2016; 188: 662-670

\section{Zusammenfassung \\ $\nabla$}

Ziel: Evaluation des erstmalig im deutschsprachigen Raum eingesetzten Ultraschall(US)-gesteuerten hoch-intensiven fokussierten Ultraschalls (HIFU) bei Patienten mit inoperablem Pankreaskarzinom zur Reduktion von Tumorvolumen und tumorbedingter Schmerzsymptomatik.

Material und Methoden: 15 Patienten mit lokal fortgeschrittenem inoperablem Pankreaskarzinom und Tumorschmerz wurden mit HIFU behandelt ( $\mathrm{n}=6$ Stadium III, $\mathrm{n}=9$ Stadium IV UICC). 13 Patienten erhielten gleichzeitig eine Standardchemotherapie. Die HIFU-Behandlung erfolgte mit dem JC HIFU System (Chongqing, China HAIFU Company) mit einer US-Vorrichtung zur Echtzeitbildgebung. Kontrolluntersuchungen (US, CT, MRT) und die klinische Evaluation durch validierte Fragebögen (NRS, BPI) wurden jeweils vor HIFU sowie in definierten Abständen bis zu 15 Monaten nach Therapie durchgeführt.

Ergebnisse: Trotz Gallengang- oder Duodenalstents (4/15) und der Ummauerung von Oberbauchgefäßen (15/15) war eine Behandlung bei allen Patienten möglich. Die mittlere Interventionsdauer 
lag bei $111 \mathrm{~min}$, die therapeutische Schallzeit bei 1103 s, die Gesamtenergie bei 386768J. Die postinterventionelle Bildgebung zeigte eine Avaskularisation der behandelten Tumorregionen mit einer signifikanten Volumenreduktion von 63,8\% nach 3 Monaten. Eine signifikante Schmerzlinderung konnte bei 12 Patienten erreicht werden (komplett $n=6$, partiell $n=6$ ).

Schlussfolgerung: Der US-gesteuerte HIFU kann bei Patienten mit lokal fortgeschrittenem inoperablem Pankreaskarzinom, sofern ein geeignetes Schallfenster vorliegt, zur lokalen Tumorkontrolle und Linderung von tumorassoziierten Schmerzen eingesetzt werden.

\section{Introduction}

$\nabla$

Approximately 16,000 patients are diagnosed with pancreatic ductal adenocarcinoma in Germany each year; men and women are equally affected. Currently surgical treatment is the only curative form of therapy. However, at the time of initial diagnosis, more than $80 \%$ of patients are inoperable with a median survival time of 4-6 months so that this tumor has the worst prognosis of all gastrointestinal tumors $[1,2]$. Palliative therapy of locally advanced pancreatic cancer involves chemotherapy or radiochemotherapy to decelerate tumor growth, avoid tumor-related complications and alleviate symptoms. Some new chemotherapy regimens such as nab-paclitaxel plus Gemcitabine, FOLFOX 6 and FOLFIRINOX have demonstrated survival advantages for patients with pancreatic cancer [3-7]; however, the 1-year survival rate is only about $18-20 \%$ and the 5-year survival rate is less than $1 \%[8]$.

An increase in pain symptoms is one of the most frequent presentations of locally advanced pancreatic cancer. Treatment includes symptomatic measures (analgesia with opioids) or celiac plexus block [9, 10]. From the palliative care point of view, the development of alternative forms of therapy is urgently required for analgesia and local tumor control.

High-intensity focused ultrasound (HIFU) is an innovative treatment option for local therapy of pancreatic cancer. Using a special transducer, HIFU generates ultrasound (US) waves which are then focused on a target within the body. The US waves create heat in the target tissue above $80^{\circ} \mathrm{C}$, resulting in coagulation necrosis ( $\bullet$ Fig. 1). In numerous studies, mainly from East Asia, HIFU has been shown to be safe, effective and practicable in its clinical application with only a few therapy-related side effects [11]. However, these are retrospective, non-randomized studies and case reports of East Asian patients [21-26]. Although these data are quite promising, it remains unclear whether the procedure will yield equally good results in Caucasian patients with inoperable pancreatic cancer. In this context we prospectively studied the efficacy and clinical advantages of HIFU as an adjunct to standard palliative therapy. The primary goals of additive HIFU therapy were sufficient local tumor control and especially pain reduction with an improvement of the clinical condition.

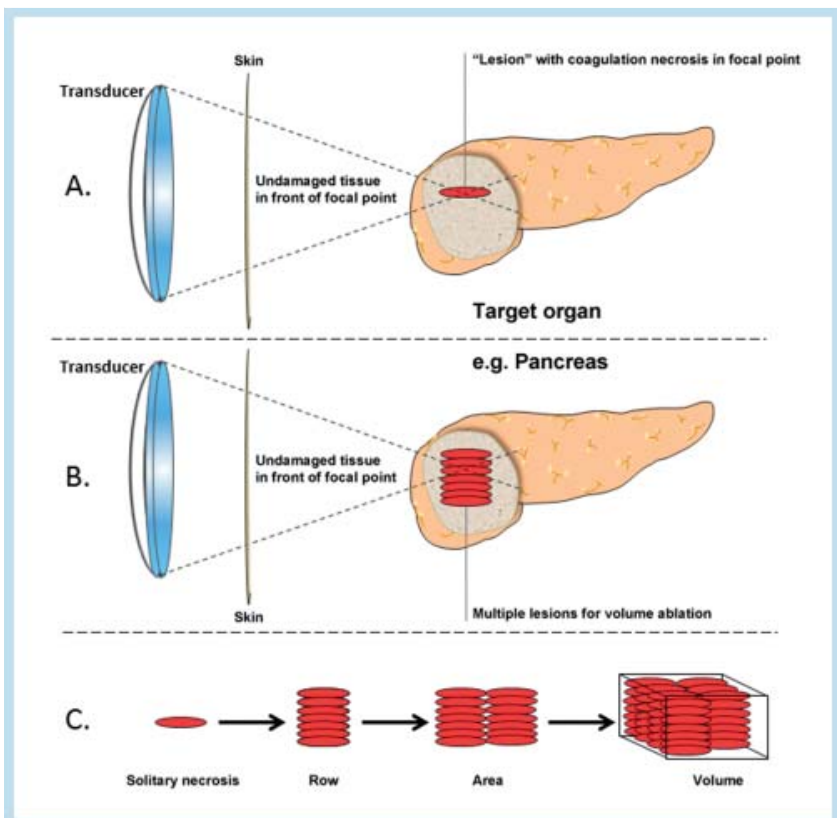

Fig. 1 HIFU mechanism. A. The extracorporeal therapeutic transducer generates a cigar-shaped focal point ( 1 - $3 \mathrm{~mm}$ wide, 8 - $15 \mathrm{~mm}$ long) with coagulation necrosis in the target region. B. Multiple lesions in the target organ. C. Linear and discoidal areas are ablated by lining up HIFU necroses until the entire tumor volume has been treated.

\section{Materials and Methods}

Patient Selection and Characteristics

15 patients ( 7 females, average age 66.9 years, 0 Table 1 ) with locally advanced inoperable pancreatic cancer were treated with US-guided HIFU. At baseline and follow-up they were prospectively evaluated using validated questionnaires (NRS, BPI) and imaging (US, MRI, CT). One patient was treated for recurrence after surgical resection and radiation therapy. All tumors were located in the direct vicinity of the stomach, intestine and/or visceral vessels (celiac trunk, splenic artery, superior mesenteric artery, common hepatic artery). An interdisciplinary tumor board determined the indication for palliative chemotherapy and/or additive HIFU treatment for each patient on an individual basis (see $\bullet$ Table 2 for inclusion/exclusion criteria).

At presentation all 15 patients exhibited tumor-related pain symptoms. 2 patients had severe pain (Numerical Rating Score - NRS 7 -10) despite opioid medication, 7 patients reported moderate pain (NRS 4-6) while taking daily basic medication (opioids $n=4$, NSAID/metamizole $n=4$ ) and 5 patients had mild pain while taking basic or on-demand medication (daily opioid intake $n=1$, daily NSAID/metamizole intake $n=2$, NSAID/metamizole as needed $n=4$ ). One patient reported no pain with daily metamizole intake.

\section{HIFU ablation}

At presentation, a medical history was obtained, a physical examination was performed and laboratory parameters were collected. On the day prior to treatment a special bowel preparation similar to colonoscopy preparation was performed. Directly prior to HIFU the skin of the patient's upper abdominal wall was shaved, degreased and degassed. 
Degassing was performed using a special device to remove even the smallest residual air bubbles from the skin pores in order to prevent skin burning.

Table 1 Patient demographics and clinical characteristics.

\begin{tabular}{|c|c|}
\hline parameter & value \\
\hline number of patients & $n=15$ \\
\hline \multicolumn{2}{|l|}{ gender } \\
\hline - male & $8(53)^{1}$ \\
\hline - female & $7(47)$ \\
\hline age (years) & $66.9 \pm 10.8(45-82)^{2}$ \\
\hline \multicolumn{2}{|l|}{$\mathrm{ECOG}^{3}$ status } \\
\hline-0 & $4(27)$ \\
\hline-1 & $6(40)$ \\
\hline-2 & $5(33)$ \\
\hline \multicolumn{2}{|l|}{ tumor location } \\
\hline - head & $7(47)$ \\
\hline - body & $5(33)$ \\
\hline - head/body & $3(20)$ \\
\hline \multicolumn{2}{|l|}{$\mathrm{UICC}^{4}$ stage } \\
\hline - stage III & $6(40)$ \\
\hline - stage IV & $9(60)$ \\
\hline \multicolumn{2}{|l|}{ metastases $(n=8)$} \\
\hline - hepatic & $7(88)$ \\
\hline - pulmonary & $1(12)$ \\
\hline \multicolumn{2}{|l|}{ CA $19-9^{5}$} \\
\hline - positive & $13(87)$ \\
\hline - negative & $2(13)$ \\
\hline vascular encasement & $15(100)$ \\
\hline \multicolumn{2}{|l|}{ biliary drainage } \\
\hline - metal stent & $2(13)$ \\
\hline - plastic stent & $2(13)$ \\
\hline \multicolumn{2}{|l|}{ pre-treatment } \\
\hline - chemotherapy & $13(87)$ \\
\hline - radiotherapy & $2(13)$ \\
\hline - non-therapeutic laparotomy & $5(33)$ \\
\hline \multicolumn{2}{|c|}{$\begin{array}{l}\text { HIFU: High-Intensity Focused Ultrasound. } \\
{ }^{1} \text { number (\%). } \\
22 \text { average } \pm \text { standard deviation (range). } \\
{ }^{3} \text { ECOG: Eastern Cooperative Oncology Group. } \\
{ }^{4} \text { UICC: Union internationale contre le cancer. } \\
{ }^{5} \text { carbohydrate antigen } 19-9 .\end{array}$} \\
\hline
\end{tabular}

Treatment utilized an approved medical device according to European standards and institutional guidelines: Model JC Focused Ultrasound Tumor Therapeutic System (Chongqing HAIFU Medical Technology, China) HIFU system equipped with a US device for real-time imaging $(1-8 \mathrm{MHz}$, MyLab70, Esaote, Genoa, Italy). A ceramic transducer (diameter $20 \mathrm{~cm}$, focal length $15 \mathrm{~cm}$, frequency $0.8 \mathrm{MHz}$ ) was used to generate the therapeutic ultrasound. The HIFU system was used in accordance with the intended purpose in compliance with the Helsinki Declaration following approval by the local ethics committee (No.302/12). This study is part of a series of studies $[12,13]$ evaluating the clinical use of HIFU in the Western medical setting and its effectiveness with respect to pain and symptom relief in combination with standard palliative therapy.

HIFU ablation was performed in a prone position under general anesthesia. Prior to the start of therapy, the tumor was first delineated and the adjacent structures and vessels identified. Then a treatment plan was developed with sagittal slices. The focus was placed in the region to be treated, and energy was applied to the dorsal tumor regions. No thermal damage was expected posterior to the focus, since the position of larger vessels (aorta, inferior vena cava) with sufficient blood flow allows heat dissipation. The final sonication at the focus $(200-400 \mathrm{~W})$ was determined individually for each patient; a safety margin of $1 \mathrm{~cm}$ to the tumor margin and existing stents was maintained. In most patients the tumor encased the superior mesenteric artery and celiac trunk. However, this was not a contraindication for HIFU treatment.

During treatment, the stomach and colon often lay in the acoustic path. To avoid possible complications, a strict bowel preparation similar to that before a colonoscopy was performed on the day prior to treatment. In particular, milk-based products were restricted and patients had to fast for approx. 12 hours prior to treatment. In addition, a gavage was used during the intervention in order to apply an anti-foaming agent (e.g. simethicone) to counteract interfering air bubbles. Moreover, a balloon filled with degassed water was placed between the patient's upper abdominal wall and the transducer to compress the stomach, push

Table 2 Selection criteria for HIFU therapy.

\section{inclusion criteria}

\section{(1) age $\geq 18$ years}

(2) inoperable pancreatic cancer with indication for palliative standard therapy

(3) written documented consent to participate in study

(4) ECOG $\leq 2$

(5) sufficient organ function:

- absolute neutrophilia $\geq 1.5 \times 10^{9} / \mathrm{L}$

- hemoglobin $\geq 8 \mathrm{~g} / \mathrm{dL}$

- platelets $\geq 75 \times 10^{9} / \mathrm{L}$

- AST/ALT $\leq 5 \times$ normal value

- gamma GT $\leq 5 \times$ normal value

- creatinine in normal range or creatinine clearance $\geq 50 \mathrm{~mL} / \mathrm{min}$

(6) tumor pain or local tumor growth

(7) sufficient sonographic visualization of tumor in prone position

as well as safe acoustic path

(8) maximum distance between skin surface and furthest focal

point in tumor, approx. $12 \mathrm{~cm}$

(9) patient suitable for anesthesia

\section{exclusion criteria}

(1) surgical resection possible

(2) patient not suitable for anesthesia

(3) ECOG $\geq 3$

(4) insufficient sonographic presentation of tumor

(e. g. postoperatively or post-radiogenic)

(5) pronounced scarring along the acoustic path

(6) very extensive distant metastasis ( $>5$ liver metastases, liver metastases $>2 \mathrm{~cm}$ ) 
the bowel away from the acoustic beam path and to maintain an optimum acoustic window. To avoid skin burning and to prevent subcutaneous edema, the skin in the acoustic pathway was regularly checked by palpation and cooled during HIFU treatment.

In the case of visible grayscale changes in the target area or after at least 50 seconds of sonication time at a specific focal point, the transducer was moved to the next focal point in order to achieve volume ablation. Toward the end of the procedure contrast-enhanced ultrasound (CEUS) was performed. If no perfusion was seen in the treated area, the therapy was considered successful; otherwise the procedure was continued.

The patients remained hospitalized for 1 - 3 days after HIFU treatment.

\section{Imaging}

Within 2 weeks prior to HIFU treatment, CT and MRI were performed to provide baseline images (Brilliance 64-slice CT scanner, Ingenia 1.5 Tesla MRI scanner, Philips Healthcare, Amsterdam, Netherlands). Directly prior to the HIFU procedure, tumor perfusion was determined using CEUS after intravenous administration of Sonovue ${ }^{\circledR}$ (Bracco, Italy).

CEUS and CT were performed during the first 24 hours after HIFU and an MRI examination was carried out within 3 days. Long-term monitoring comprised CT/MRI/CEUS examinations after 6 weeks, 3 months and then in threemonth intervals. The tumor ablation rate (\%) was calculated as the ratio between the avascularized (ablated) volume and the total volume; the volume reduction (\%) was determined after 6 weeks and 3 months. Therapy-related side effects and complications were recorded [14].

\section{Evaluation of Pain Reduction}

Changes in pain symptoms and medication were recorded during follow-up examinations. Measurement tools included: NRS (Numerical Rating Score 0 - 10), scores for pain intensity (current, average, minimum and maximum pain) from the BPI questionnaire (Brief Pain Inventory; intensity levels $0-10)$. In both questionnaires 0 means "no pain" and 10 represents the "worst pain imaginable" [15]. An NRS reduction to $\leq 1$ was defined as complete pain response. Partial pain reduction was related to an NRS reduction by $\geq 2$ [16 - 18].

\section{Statistical Analysis}

The collected data were analyzed using Stata Version 13.1 (Stata Corp, Lakeway, College Station, Texas USA). Primary statistical analysis was performed using a mixed panel data model. Model robustness was verified with a non-parametric Skilling-Mack test for unbalanced panel data. A p-value of less than 0.05 was considered statistically significant.

\section{Results \\ $\nabla$}

\section{HIFU Treatment}

In accordance with inclusion and exclusion ( $\bullet$ Table 2 ), one third of the evaluated patients were accepted criteria for treatment. The pancreatic tumor could be sufficiently visualized sonographically in all of these patients. Fourteen patients were treated with US-guided HIFU in a single session, and one was treated in two sessions. Seven months after the first HIFU treatment, the patient treated twice demonstrated tumor regrowth left lateral to the post-therapeutic necrotic cavity in the pancreatic body. This recurrence was treated again with HIFU. The time interval between the initial diagnosis and HIFU therapy averaged $9 \pm 8.1$ months. In each patient an attempt was made to significantly ablate the tumor. However, by maintaining safety distances from adjacent high-risk structures (stomach, bowel, vessels, stents), some vital tumor tissue remained in the margin area. Given a safety margin of $0.5-1 \mathrm{~cm}$ with respect to vessels, no occlusion of larger vessels occurred ( $\bullet$ Fig. 3 ).

The therapeutic parameters as well as the duration of intervention are summarized in 0 Table 3. In 10 of 15 patients grayscale changes in the $\mathrm{B}$-mode image (distinct $\mathrm{n}=5$, moderate $n=5$ ) were observed within treated regions during the HIFU procedure. In 5 cases, no visible grayscale changes were seen in the treated tumor areas, even though the CEUS examination performed during HIFU showed them as avascularized.

\section{Follow-up Imaging}

Within 24 hours after HIFU, treated tumor regions exhibited avascularization on CEUS ( $\bullet$ Fig. 2). CT ruled out severe therapy-related complications. Post-intervention contrastenhanced examinations (MRI, CEUS) showed devascularization of the ablated area ( $\bullet$ Fig. 2, 3). Short-term MRI followup did not show any significant change in tumor volume ( $30.3 \pm 18.8 \mathrm{ml}$ vs. $31.4 \pm 18.3 \mathrm{ml}$ at baseline). The tumor ablation rate was $53.1 \%$ (range $33.7-72.3 \%$ ). The average reduction in tumor volume was approx. $25.9 \%$ after 6 weeks and $63.8 \%$ after 3 months ( $\bullet$ Fig. 2,4 ). Tumor growth outside the treated region was observed in 2 patients. One of them was treated again with HIFU; in the other case, the tumor had infiltrated the intestinal wall.

\section{Pain Reduction}

Up to 24 hours post-intervention 10 of the 15 patients reported minor $(n=4)$ to severe $(n=6)$ upper abdominal pain. 2-3 days after a single HIFU treatment, 12 patients reported pain reduction ( $\bullet$ Fig. 5,6 ). 6 of these patients were pain-free (NRS $\leq 1$ ), while the other 6 patients experienced significant pain reduction (NRS reduction $\geq 2$ ).

During the 6-week follow-up, the originally prescribed opioid analgesics were able to be discontinued as basic medication in 2 of 7 patients. The remaining 5 patients reported an NRS decrease of $\geq 2$ with $(n=2)$ or without $(n=3)$ an opioid dose reduction. None of the patients reported severe pain. 4 patients indicated moderate pain (opioid with dose reduction $n=2$, NSAID/metamizole on-demand $n=3$ ). 6 patients reported minor pain (metamizole basic medication $n=1$, NSAID/metamizole on-demand $n=4$, no medication $n=1)$. In the case of 3 patients with progressive hepatic/peritoneal metastasis, there was no change in the pain score after HIFU. Tumor-related pain in 5 patients with peritoneal carcinosis and liver metastases responded well to HIFU.

\section{Side Effects}

Transient (sub)cutaneous edema of the proximal upper abdominal wall was observed in 9 of 15 patients. In one patient there was superficial skin burning in the region of the 

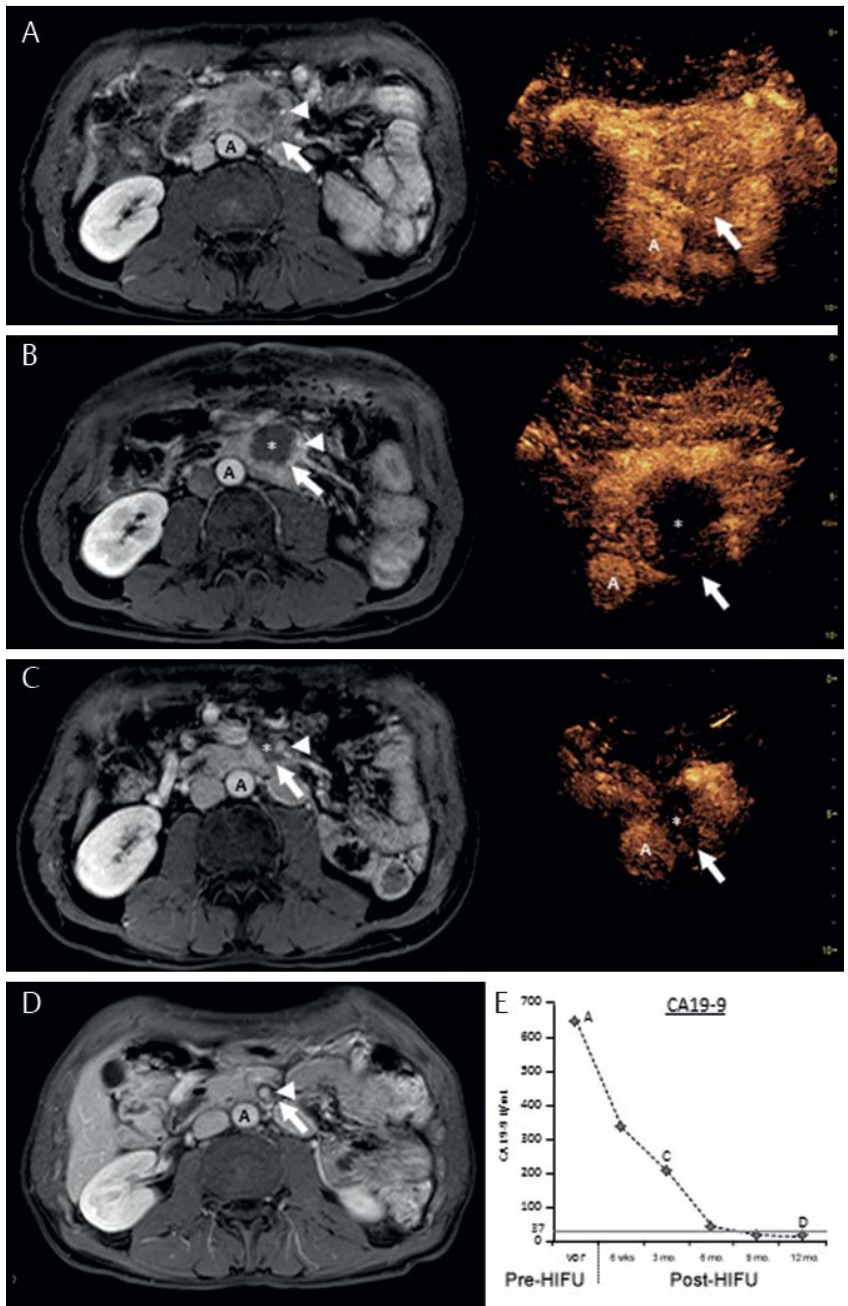

Fig. 2 A 56-year-old male patient with inoperable locally progressive adenocarcinoma of the pancreatic head and pronounced tumor pain was treated in our clinic with HIFU after the first FOLFIRINOX cycle had been completed. Two days after HIFU treatment the patient was pain-free (NRS = 8 pre-HIFU; NRS $=1$ one week post-HIFU). Contrast-enhanced transverse MR and US images of the treated tumor are presented as follows A-D. A. Pancreatic cancer prior to HIFU therapy with encasement of superior mesenteric artery. CA 19-9: $648.8 \mathrm{U} / \mathrm{mL}$. B. Recognizable ablation cavity $\left({ }^{*}\right)$ without contrast enhancement 1 day post-intervention. C. Reduction of tumor volume by $90.1 \%$ in 3-month follow-up. CA19 - 9: 205.6 $\mathrm{U} / \mathrm{mL}$. D. Tumor remission 12 months post-HIFU CA19-9: $15.8 \mathrm{U} / \mathrm{mL}$. E. Tumor marker CA19 - 9 over the course of 12 months (reference range 2 $37 \mathrm{Um} / \mathrm{L}$; the gray line shows the upper normal value). A: Aorta. ${ }^{*}$ ablation area. Arrow: pancreatic cancer. Arrowheads: superior mesenteric artery.

navel (degree IIa) which healed spontaneously within 3 weeks. Transient induration of subcutaneous adipose tissue in the proximal upper abdominal wall was observed in one patient. Three patients experienced a temporary increase in pancreatic lipase after HIFU, but without clinical signs of pancreatitis. Severe or long-lasting side effects were not observed.

\section{Discussion}

$\nabla$

There is an urgent need for locally effective therapies for inoperable locally advanced pancreatic cancer which not only achieve sufficient tumor control, but also provide palliation
Table 3 Therapeutic parameters.

\begin{tabular}{|c|c|}
\hline \multicolumn{2}{|c|}{ Treatment time } \\
\hline [min] & $110.5 \pm 30.7(66-190)$ \\
\hline \multicolumn{2}{|c|}{ Sonication time } \\
\hline [s] & $1103 \pm 456(548-2452)$ \\
\hline [min] & $18.4 \pm 7.6(9.1-40.9)$ \\
\hline \multicolumn{2}{|l|}{ Total energy } \\
\hline [J] & $386768 \pm 173256(168350-851200)$ \\
\hline \multicolumn{2}{|c|}{ Output range } \\
\hline$[\mathrm{W}]$ & $80-400$ \\
\hline \multicolumn{2}{|c|}{ Average output } \\
\hline$[\mathrm{W}]$ & $344 \pm 69(200-400)$ \\
\hline \multicolumn{2}{|c|}{ Grayscale change } \\
\hline Significant & $5(33 \%)$ \\
\hline Moderate & $6(40 \%)$ \\
\hline No change & $4(27 \%)$ \\
\hline
\end{tabular}

of tumor-related pain symptoms while improving the patient's quality of life. In recent years local therapy of inoperable pancreatic cancer has included procedures such as cryotherapy, radiofrequency ablation and microwave therapy [19-22]. However, these approaches range from minimally invasive to invasive, since local energy can be generated only after one or more needles have been inserted into the human body with an increased risk of injury to adjacent structures (stomach, bowel, vessels). Currently there is no established strategy for local treatment of pancreatic cancer. Therefore, HIFU could be employed in particular to treat progressive tumor-related symptoms while providing additional clinical benefits. HIFU is an innovative procedure allowing targeted non-invasive thermal ablation of tissues accessible by US without requiring the insertion of needles, electrodes or probes. In addition to heat generation with coagulation necrosis [23-25], the action mechanisms also include cavitation and potential immunological effects [26-28].

MRI or sonography can be used to guide HIFU. Although MRI achieves very good anatomical resolution, the realtime resolution is limited. MRI-guided equipment is therefore generally used to treat uterine fibroids and bone tumors. In contrast, diagnostic US provides real-time imaging. Equipment design allows the use of larger and more powerful transducers, generating higher temperatures in the target region, thus allowing faster treatment. Although pancreatic cancer can be difficult to identify sonographically and therefore is often overlooked, the tumors could be visualized in all patients during US evaluation and subsequently treated with HIFU. An advantage of US-guided systems is that a tumor that can be identified sonographically can also be treated with HIFU. In contrast, MRI may visualize a tumor that however cannot be treated with ultrasound due to overlying bowel gas or bone.In addition, USguided equipment makes real-time imaging possible. Although respiration results in less movement of the pancreas, displacement of ventrally located structures such as the stomach and colon often occurs. Air in the stomach or colon must be identified promptly in order to interrupt the therapy if necessary and select a different acoustic window. Using sonography for intrainterventional monitoring is more difficult since only about a third of patients exhibit hyperechoic changes in the treated region. The amount of 

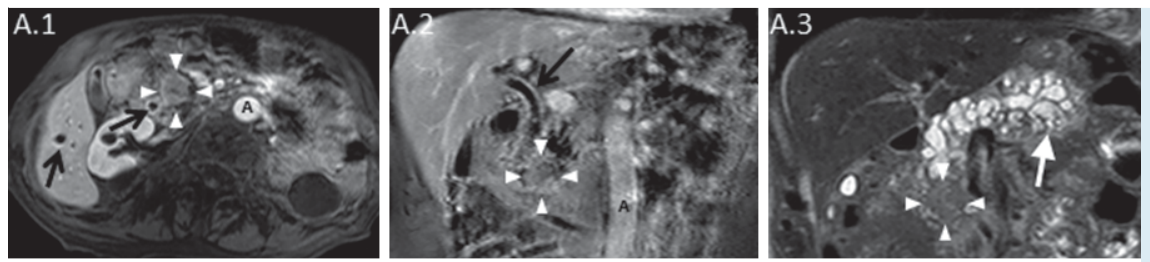

Fig. 3 An 82-year-old male patient with inoperable cancer of the pancreatic head within the cystically transformed pancreas (white arrow) and enclosed PTCD (percutaneous transhepatic cholangiography and drainage, Yamacawa drainage) was treated with HIFU. White arrowheads indicate the tumor. A.1, B.1 Transverse
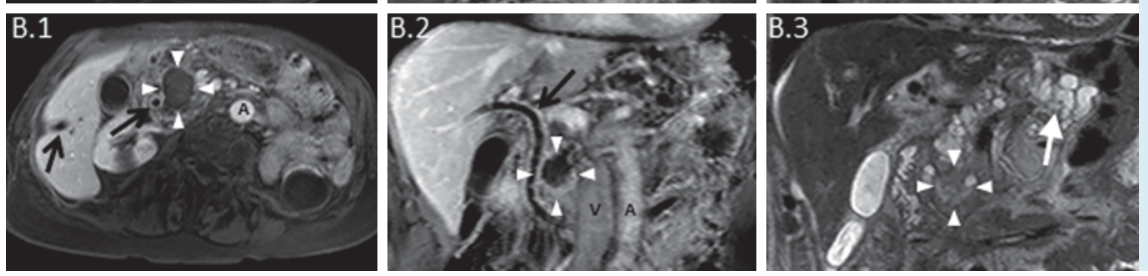
contrast-enhanced MRI images before and after HIFU. A.2, B.2 Coronal contrast-enhanced MRI images before and after HIFU. A.3, B.3 Coronal T2-weighted MRI images before and after HIFU. A: Aorta. V: inferior vena cava.

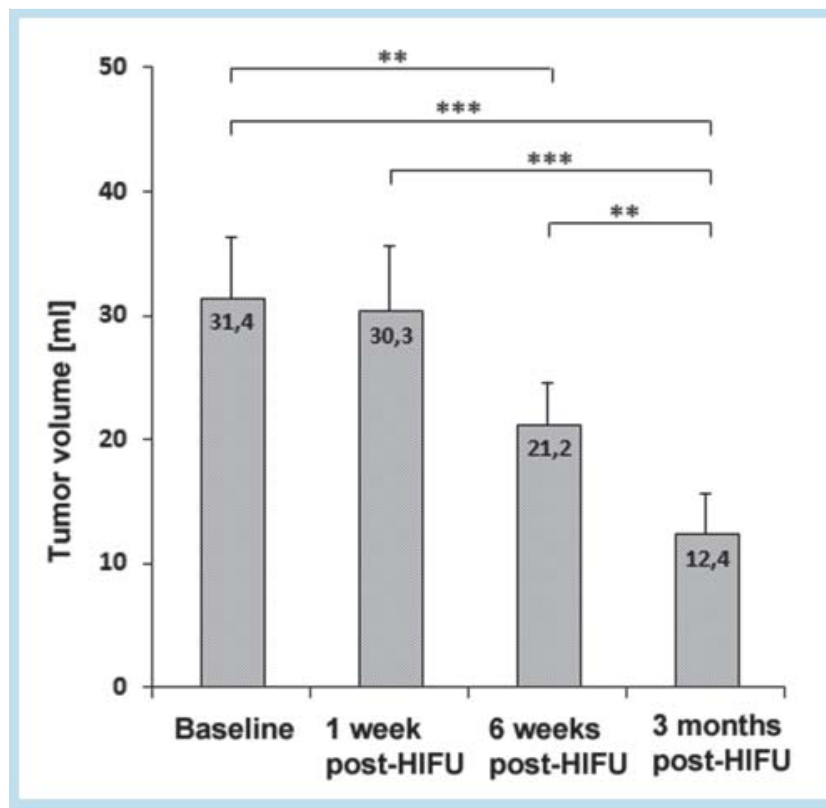

Fig. 4 Change in tumor volume post-HIFU. Tumor volumes (in $\mathrm{ml}$ ) are shown with mean and standard deviation. Over time there was an average volume reduction ( $n=15)$ of $26 \%$ after 6 weeks and $64 \%$ after 3 months.

applied energy (min. $50 \mathrm{~s}$ sonication time per focal point) or intermittently performed CEUS showing devascularization of the treated area is used to assess sufficient treatment at a focal point ( 0 Fig. 2, 3). Most of our patients were treated at 400 watts. The energy was reduced to 200 watts in the direct vicinity of vessels, the bowel or stents ( $\bullet$ Fig. 3). Even though US-guided HIFU devices are more efficient than MRI-guided equipment, the advantage of real-time imaging is much more important in the case of pancreatic cancer. Except for a retrospective report from Italy including 6 patients [29], all data regarding the feasibility and safety of US-guided HIFU therapy for inoperable pancreatic cancer come from East Asia and generally present retrospective observations [16 - 18, 29-34]. Meanwhile numerous Chinese studies were summarized in an overview article that reported on HIFU treatment of a total of 3022 patients with inoperable pancreatic cancer during the previous 15 years [35]. However, more than $70 \%$ of the publications are available only in Chinese, and more than $90 \%$ of the studies were carried out in China, Korea and Japan. To

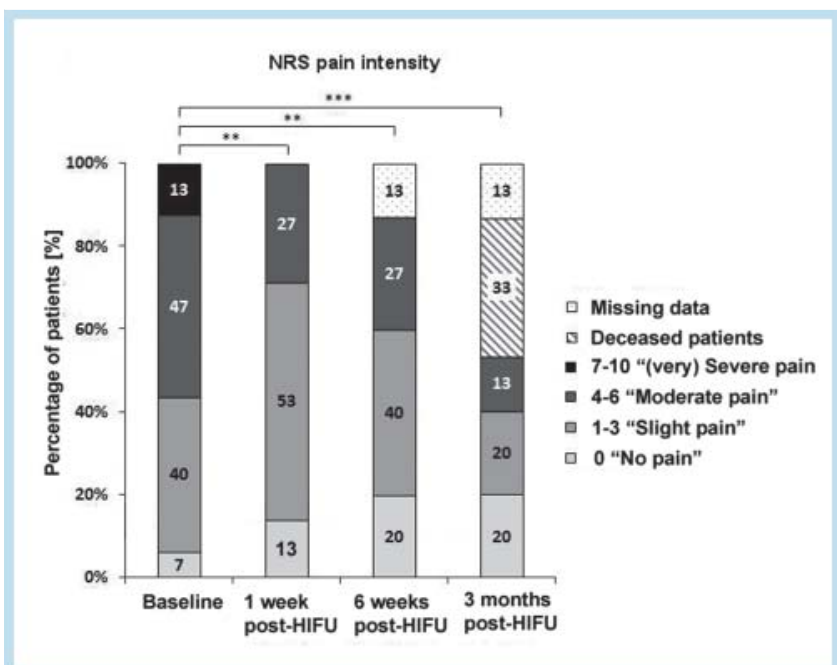

Fig. 5 Pain reduction post-HIFU; evaluation with NRS (Numerical Rating Score). Percentage of patients in the different NRS categories (0: no pain; 7-10 (very) severe pain) before HIFU and 1 week, 6 weeks and 3 months after HIFU. Missing data as well as the percentage of deceased patients are also shown.

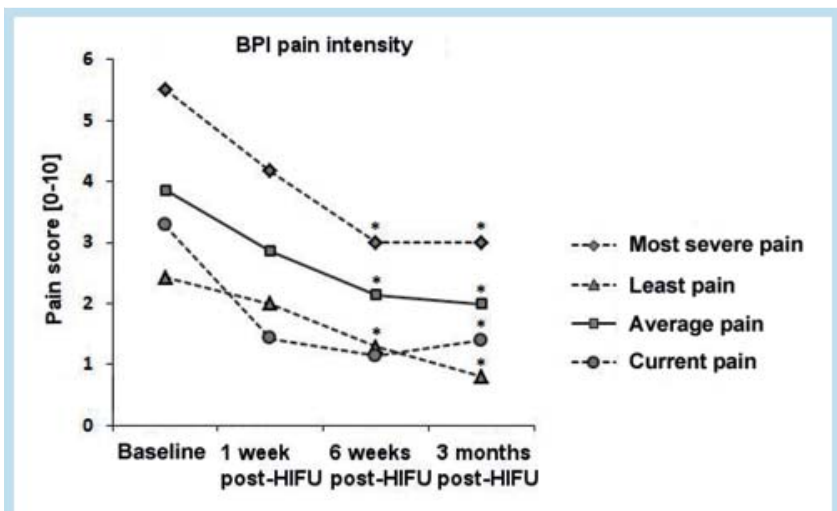

Fig. 6 Change in pain intensity post-HIFU; evaluation with BPI (Brief Pain Inventory).

date, there have been no prospective randomized studies according to European scientific standards regarding the application and effectiveness of HIFU with respect to local tumor control, pain and symptom reduction, progression- 
free survival and overall survival in combination with standard palliative therapy.

Therefore, we prospectively evaluated the effectiveness and clinical advantages of additive HIFU treatment for our Caucasian patient cohort. We used standardized questionnaires (NRS, BPI) and imaging (US, CT, MRI) for evaluation. Immediately after HIFU all patients exhibited therapy-based avascularization of the treated tumor regions. In the follow-up, significant tumor volume reduction was observed in 13 of 15 patients ( 0 Fig. 2,4 ). The tumor volume reduction was $90.1 \%$ after 3 months for the first patient treated with HIFU ( $\odot$ Fig. 2) and the 21-month follow-up indicated continued tumor remission. This emphasizes the advantages of additive HIFU ablation, not only regarding significant tumor reduction, but also with respect to slowing the progression of tumor-related local symptoms which is frequently not possible using chemotherapy alone. Sufficient local tumor control could also be achieved even in the case of advanced hepatic, pulmonary or peritoneal metastasis. Since HIFU treatment does not interact with standard palliative therapy and is a low-risk procedure with only a few transient side effects, chemotherapy can be continued without interruption.

Tumor-related pain is one of the most frequent symptoms in patients with pancreatic cancer, occurring in more than $80 \%$ of cases [2]. In advanced disease stages, opioid analgesics are frequently used, resulting in side effects such as nausea, vomiting, constipation and drowsiness. Local therapies such as percutaneous celiac plexus block [36] or radiotherapy $[37,38]$ have a limited long-term effect on pain control. In our patient cohort, 12 of 15 patients required daily basic medication at baseline, 7 of whom received opioids. After HIFU $80 \%$ of patients $(n=12)$ achieved significant pain reduction within 24-48 hours after treatment. Energy application in the direct vicinity of the celiac trunk with its pain fibers likely contributes to the analgesic effect resulting from damage to the celiac plexus [13]. Data published to date report comparable pain reduction in $84.7 \%$ of patients [16, 18, 29, $31-34]$. For 10 patients who needed daily long-term medication to control their pain prior to HIFU, their dosage of medication was reduced by at least half after the intervention. The previous opioid analgesia of 2 patients was even able to be completely eliminated. The pain reduction continued up to the follow-up after 3 months ( $\bullet$ Fig. 5,6 ). The reduction in pain intensity consequently led to an improvement of patient quality of life. Prior to HIFU one third of the patients reported mild pain (WHO level I), and half of the patients did not require opioids. Nevertheless the observed pain reduction follow- ing from HIFU is distinct and considerable; therefore the analgesic effect should be evaluated in a larger study with patients taking basic opioid medication.

In addition to local tumor control and reduction of tumorrelated pain, HIFU can have a positive effect on the progression-free and overall survival of patients [39]. A survival advantage of local tumor control can only be assumed, based on published data. Asian studies have reported a median overall survival of $6-11$ months and a median progression-free time of 5 to 8.4 months [16 - 18]. Our observational study was not designed to evaluate survival rate since there were no control group data available. Nevertheless, a median overall survival of 13.3 months - comparable to published results - could be shown [30, 39] ( $\odot$ Fig. 7). During the 15 -month observation period, 8 patients died of progressive hepatic, peritoneal or pulmonary metastasis, and 2 patients died due to advanced bowel infiltration.

Even though HIFU is considered a low-risk procedure with generally few and rare side effects compared to other therapies, various HIFU-related complications have been described [40]. In particular, organs with high acoustic absorption such as the skin and gastrointestinal tract have an increased risk of thermal damage. However, regular cooling of the skin and stringent bowel preparation can efficiently prevent these complications. Superficial skin and subcutaneous tissue injury has been reported in $3.1 \%$ of cases. Pancreatitis (1.9\%) and pancreoprivic diabetes mellitus (1.3\%) represent additional possible organ-specific complications [40].

One of our patients suffered superficial skin burning in the region of the navel; another experienced induration of subcutaneous adipose tissue; both healed within 3-6 weeks without special therapy. In addition up to 24 hours post-intervention, upper abdominal pain $(n=10)$ and cutaneous edema of the abdominal wall $(n=9)$ were observed in the majority of patients. Severe or long-lasting side effects were not observed.

\section{Conclusion}

US-guided HIFU therapy represents a safe and effective ablation procedure to reduce tumor volume in patients with inoperable pancreatic cancer. The use of HIFU can result in a significant reduction in tumor-related pain. Further prospective controlled, randomized studies with larger patient cohorts are required to evaluate the long-term effectiveness, especially with respect to progression-free and overall survival.

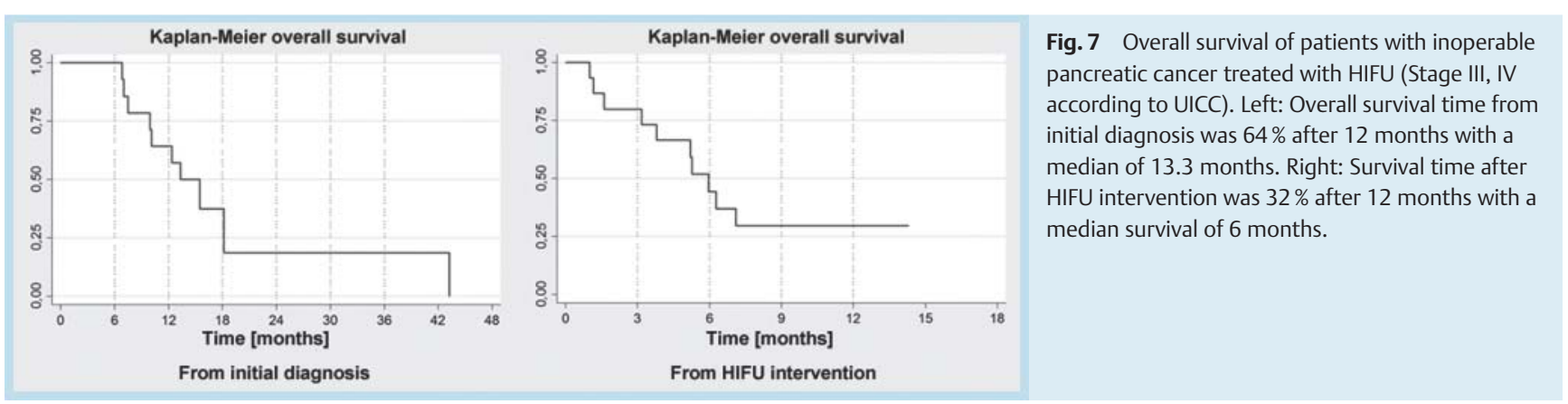


Clinical Relevance of the Study

1. Local US-guided HIFU treatment for inoperable pancreatic cancer in combination with standard palliative therapy is a safe and effective ablation procedure, given the presence of a suitable acoustic window.

2. With a low rate of side effects, this innovative therapy offers the additional clinical benefit of alleviating patient discomfort and symptoms as a result of pain reduction and possibly a decrease in tumor size.

\section{Affiliations}

1 Department of Radiology, Medical School \& Hospital, University of Bonn

2 Department of Palliative Medicine, Medical School \& Hospital, University of Bonn

3 Department of General Practice and Family Medicine, Medical School \& Hospital, University of Bonn

${ }^{4}$ Center for Rare Diseases Bonn (ZSEB), Medical School \& Hospital, University of Bonn

${ }^{5}$ Chongqing Key Laboratory of Ultrasound in Medicine and Engineeríng, Chongqing Medical University, China
}

\section{References}

1 Ryan DP, Hong TS, Bardeesy N. Pancreatic adenocarcinoma. The New England journal of medicine 2014; 371: 1039-1049

2 Seufferlein T, Porzner M, Heinemann $V$ et al. Ductal pancreatic adenocarcinoma. Deutsches Arzteblatt international 2014; 111: 396-402

3 Aaronson NK, Ahmedzai S, Bergman B et al. The European Organization for Research and Treatment of Cancer QLQ-C30: a quality-of-life instrument for use in international clinical trials in oncology. J Natl Cancer Inst 1993; 85: 365 - 376

4 Conroy $T$, Desseigne $F$, Ychou $M$ et al. FOLFIRINOX versus gemcitabine for metastatic pancreatic cancer. The New England journal of medicine 2011; 364: 1817 - 1825

5 Ghosn M, Farhat F, Kattan J et al. FOLFOX-6 combination as the firstline treatment of locally advanced and/or metastatic pancreatic cancer. Am J Clin Oncol 2007; 30: 15 - 20

6 Trouilloud I, Dupont-Gossard AC, Malka D et al. Fixed-dose rate gemcitabine alone or alternating with FOLFIRI.3 (irinotecan, leucovorin and fluorouracil) in the first-line treatment of patients with metastatic pancreatic adenocarcinoma: an AGEO randomised phase II study (FIRGEM). European journal of cancer 2014; 50: 3116-3124

7 Von Hoff DD, Ervin T, Arena FP et al. Increased survival in pancreatic cancer with nab-paclitaxel plus gemcitabine. The New England journal of medicine 2013; 369: 1691 - 1703

8 Sultana A, Smith CT, Cunningham D et al. Meta-analyses of chemotherapy for locally advanced and metastatic pancreatic cancer. Journal of clinical oncology: official journal of the American Society of Clinical Oncology 2007; 25: 2607-2615

9 Reddy SK, Elsayem A, Talukdar R. Supportive Care: Symptom Management. Pancreatic Cancer 2005: 479-498

10 Yan BM, Myers RP. Neurolytic celiac plexus block for pain control in unresectable pancreatic cancer. The American journal of gastroenterology 2007; 102: $430-438$

11 Wu F, Wang ZB, Chen WZ et al. Extracorporeal high intensity focused ultrasound ablation in the treatment of 1038 patients with solid carcinomas in China: an overview. Ultrasonics sonochemistry 2004; 11 : $149-154$

12 Marinova $M$, Rauch $M$, Mücke $M$ et al. High-intensity focused ultrasound (HIFU) for pancreatic carcinoma: evaluation of feasibility, reduction of tumour volume and pain intensity. Eur Radiol 2016, ahead of print; DOI: 10.1007/s00330-016-4239-0

13 Marinova M, Strunk HM, Rauch $M$ et al. Use of high-intensity focused ultrasound (HIFU) in inoperable pancreatic cancer for tumor pain relief: evaluation with pain sensation scale (SES). Der Schmerz 2016, ahead of print

14 Sacks D, McClenny TE, Cardella JF et al. Society of Interventional Radiology clinical practice guidelines. Journal of vascular and interventional radiology: JVIR 2003; 14: S199-S202
15 Radbruch L, Loick G, Kiencke P et al. Validation of the German version of the Brief Pain Inventory. Journal of pain and symptom management 1999; 18: $180-187$

16 Li PZ, Zhu SH, He W et al. High-intensity focused ultrasound treatment for patients with unresectable pancreatic cancer. Hepatobiliary \& pancreatic diseases international: HBPD INT 2012; 11: 655 - 660

17 Wang $K$, Chen Z, Meng $Z$ et al. Analgesic effect of high intensity focused ultrasound therapy for unresectable pancreatic cancer. International journal of hyperthermia: the official journal of European Society for Hyperthermic Oncology, North American Hyperthermia Group 2011; 27: $101-107$

18 Gao HF, Wang K, Meng ZQ et al. High intensity focused ultrasound treatment for patients with local advanced pancreatic cancer. Hepato-gastroenterology 2013; 60: 1906-1910

19 Keane MG, Bramis K, Pereira SP et al. Systematic review of novel ablative methods in locally advanced pancreatic cancer. World journal of gastroenterology: WJG 2014; 20: 2267 -2278

20 Ierardi AM, Lucchina $N$, Petrillo $M$ et al. Systematic review of minimally invasive ablation treatment for locally advanced pancreatic cancer. La Radiologia medica 2014; 119: 483 - 498

21 Salgado S, Sharaiha R, Gaidhane M et al. Ablation therapies for pancreatic cancer: an updated review. Minerva Gastroenterol Dietol 2014; 60: $215-225$

22 Dimitrov D, Feradova H, Gincheva $D$ et al. Ablative techniques in advanced pancreatic cancer: do they affect the quality of life?-Review. J Pancreas (online) 2015; 16: 425-431

23 Sofuni A, Moriyasu F, Sano T et al. The current potential of high-intensity focused ultrasound for pancreatic carcinoma. Journal of hepatobiliary-pancreatic sciences 2011; 18: 295-303

24 Hynynen $K$, Lulu BA. Hyperthermia in cancer treatment. Investigative radiology 1990; 25: $824-834$

25 Hwang JH, Wang YN, Warren C et al. Preclinical in vivo evaluation of an extracorporeal HIFU device for ablation of pancreatic tumors. Ultrasound in medicine \& biology 2009; 35: 967 - 975

$26 \mathrm{Wu} F$, Zhou L, Chen WR. Host antitumour immune responses to HIFU ablation. International journal of hyperthermia: the official journal of European Society for Hyperthermic Oncology, North American Hyperthermia Group 2007; 23: 165 - 171

$27 \mathrm{Hu}$ Z, Yang XY, Liu Y et al. Investigation of HIFU-induced anti-tumor immunity in a murine tumor model. Journal of translational medicine 2007; $5: 34$

28 Liu F, Hu Z, Qiu L et al. Boosting high-intensity focused ultrasound-induced anti-tumor immunity using a sparse-scan strategy that can more effectively promote dendritic cell maturation. Journal of translational medicine 2010; 8: 7

29 Orsi F, Zhang L, Arnone P et al. High-intensity focused ultrasound ablation: effective and safe therapy for solid tumors in difficult locations. American journal of roentgenology 2010; 195: W245-W252

30 Sung HY, Jung SE, Cho SH et al. Long-term outcome of high-intensity focused ultrasound in advanced pancreatic cancer. Pancreas 2011; 40: $1080-1086$

31 Wang $K$, Zhu H, Meng $Z$ et al. Safety evaluation of high-intensity focused ultrasound in patients with pancreatic cancer. Onkologie 2013; 36: $88-92$

32 Wu F, Wang ZB, Zhu H et al. Feasibility of US-guided high-intensity focused ultrasound treatment in patients with advanced pancreatic cancer: initial experience. Radiology 2005; 236: 1034-1040

33 Xiong $L L$, Hwang $J H$, Huang XB et al. Early clinical experience using high intensity focused ultrasound for palliation of inoperable pancreatic cancer. JOP: Journal of the pancreas 2009; 10: 123-129

34 Zhao H, Yang G, Wang D et al. Concurrent gemcitabine and high-intensity focused ultrasound therapy in patients with locally advanced pancreatic cancer. Anti-cancer drugs 2010; 21: 447-452

35 Zhou $Y$. High-intensity focused ultrasound treatment for advanced pancreatic cancer. Gastroenterology research and practice 2014; 2014: 205325

36 Polati E, Finco G, Gottin L et al. Prospective randomized double-blind trial of neurolytic coeliac plexus block in patients with pancreatic cancer. The British journal of surgery 1998; 85: 199-201 
37 Andre T, Balosso J, Louvet $C$ et al. Combined radiotherapy and chemotherapy (cisplatin and 5-fluorouracil) as palliative treatment for localized unresectable or adjuvant treatment for resected pancreatic adenocarcinoma: results of a feasibility study. International journal of radiation oncology, biology, physics 2000; 46: 903-911

38 Ceha HM, van Tienhoven G, Gouma DJ et al. Feasibility and efficacy of high dose conformal radiotherapy for patients with locally advanced pancreatic carcinoma. Cancer 2000; 89: $2222-2229$
39 Vidal-Jove J, Perich E, del Castillo MA. Ultrasound Guided High Intensity Focused Ultrasound for malignant tumors: The Spanish experience of survival advantage in stage III and IV pancreatic cancer. Ultrasonics sonochemistry 2015; 27: 703-706

$40 \mathrm{Yu} T$, Luo J. Adverse events of extracorporeal ultrasound-guided high intensity focused ultrasound therapy. PloS one 2011; 6: e26110. DOI: $10.1371 /$ journal.pone.0026110 